\title{
Comparing Efficacy of Shoulder Ultrasound and Magnetic Resonance Imaging in Shoulder Impingement
}

\begin{abstract}
Medhat M. Refaat ${ }^{\mathrm{a}}$, Ahmed Torky ${ }^{\mathrm{a}}$, Waleed Salah El Deen ${ }^{\mathrm{b}}$, Sally Soliman ${ }^{\mathrm{a}}$
a Department of Radiodiagnosis, Benha faculty of medicine, Benha University, Egypt. b Department of Rheumatology and Physical medicine, Benha faculty of medicine, Benha University, Egypt

Correspondence to: Sally Soliman, Department of Radiodiagnosis, Benha faculty of medicine, Benha University, Egypt.

Email:

sally_diab5432@yahoo.com

Received: 6 November 2020

Accepted: 13 November 2020

Abstract

Background: Shoulder impingement syndrome is the most frequent cause of shoulder pain. Ultrasonography (US) and magnetic resonance imaging (MRI) have pivotal role for diagnosing shoulder impingement and rotator cuff pathologies. There are several advantages of US; being a dynamic form of imaging as compared to the static MRI, it is portable, better tolerated by the patient, less time consuming, less expensive and more readily available. Aim: The aim of this study is to compare the efficacy of US and MRI in diagnosis of shoulder impingement, using MRI as a reference standard. Methods: Thirty patients with clinical diagnosis of subacromial impingement were imaged by both US and MRI in the department of Radiology at Benha University Hospitals. The findings of the 2 modalities were recorded and compered. Results: There was no statistically significant difference found between MRI \& US regarding supraspinatus tendon pathology and non-rotator cuff pathology (P> 0.05). US showed a sensitivity, specificity, PPV, NPV and accuracy of $100 \%$ for each in diagnosing full thickness tear of supraspinatus tendon using MRI as reference. For partial thickness tears, it showed a sensitivity of $80 \%$, specificity of $95 \%$, PPV of $88.9 \%$, NPV of $90.5 \%$ and accuracy of $90 \%$. Conclusion: US is comparable to MRI in evaluation of shoulder impingement syndrome and rotator cuff tears, particularly more accurate in full thickness tears. Since US is less expensive and more available, with advantage of dynamic real time assessment, it could be used as the first line investigation in patients presenting with shoulder pain.
\end{abstract}

Key words: Ultrasonography, Magnetic resonance imaging, Shoulder impingement, Rotator cuff disorders 



\section{List of Abbreviations:}

SIS: Shoulder impingement syndrome

US: Ultrasonography

MRI: Magnetic resonance imaging

PPV: Positive predictive value

NPV: Negative predictive value

\section{Introduction}

Shoulder impingement syndrome (SIS) is the most common disorder of the shoulder ${ }^{1}$, as it is a common diagnosis for patients who present with pain and dysfunction of the shoulder 2.

The etiology of this condition is recognized as being multifactorial resulting from interplay of intrinsic and extrinsic factors ${ }^{3}$, but the cause of most impingements is the narrowing of the supraspinatus outlet and this phenomenon can be described as outlet impingement ${ }^{4}$.

One suggested hypothesis of the underlying pathology is chronic entrapment of the subacromial bursa and supraspinatus tendon between the humeral head and coracoacromial $\operatorname{arch}^{5}$. The coracoacromial arch is an osseoligamentous structure consisting of the undersurface of the acromion, acromioclavicular joint, the coracoacromial ligament and the coracoid process of the scapula ${ }^{6}$. Thus, the anatomic variations in the shape and slope of the acromion can lead to a predisposition to impingement syndrome, as can prominence of the acromioclavicular joint ${ }^{7}$.

As a result, the space for the bursa and tendon is reduced, and repeated trauma to these structures leads to bursitis ${ }^{8}$ and a variety of rotator cuff pathologies, such as tendinosis, and partial and full-thickness tendon tears ${ }^{9}$. About $95 \%$ of rotator cuff tears result from impingement ${ }^{7}$.

It is diagnosed clinically based on history and clinical signs as Neer impingement sign and Hawkins-Kennedy impingement sign. Imaging is helpful adjunct for confirming the diagnosis ${ }^{10}$.

When assessing a patient with signs of impingement, it is important to ascertain the integrity of the rotator cuff and the extent of the tear, if there is one. This information allows the surgeons to plan a strategy for further management of the patient ${ }^{11}$.

Due to technical improvements and advances in the understanding of anatomic and pathologic characteristics of the rotator cuff, ultrasound (US) and magnetic resonance imaging (MRI) have gradually replaced the role of arthrography for diagnosing rotator cuff tear during the past decade $^{12}$. Both methods are now accepted as routine diagnostic regimens ${ }^{13}$. Both US and MRI carry certain advantages: non-radiative, 
noninvasive, and as well as offering a multiplanar imaging ${ }^{14}$. Both of them can be considered highly specific tools for the diagnosis of rotator cuff disorders, while being highly sensitive mostly for fullthickness rotator cuff tears. However, the overall sensitivity of these tools is lower for the characterization of partial rotator cuff tears and tendinopathy ${ }^{15}$.

There are several advantages of US over MRI. Ultrasonography has the benefit of being a dynamic form of imaging as compared to the static MRI. It is portable and better tolerated by the patient and allows interaction with the patient, who can point at the symptomatic area, which will optimize the diagnostic yield ${ }^{11}$. US is also less time consuming and less expensive and more readily available in secondary and tertiary care than MRI ${ }^{16}$.

On the other hand, the ability of ultrasound waves to penetrate tissues is limited, and this technique requires very experienced examiners ${ }^{17}$.

MRI can be used to verify all types of damage to soft tissues, even tissues in less accessible locations or tissues that already have degenerative or post-injury deformations. MRI owes its ability to adequately visualize a range of different tissues to a broad flexibility of approaches in obtaining images using many different settings (induction coil, position of examined body parts, contrast enhancement, weighting, sequences with fat suppression or $3 \mathrm{D}$ reconstruction) ${ }^{17}$.

The disadvantages of MRI include its very high price, the long duration of an exam, and possible artifacts on images obtained from patients with metal implants ${ }^{17}$.

This work aimed to compare the efficacy of ultrasound and magnetic resonance imaging in diagnosis of shoulder impingement syndrome, using MRI as a reference standard.

\section{Patients and methods:}

This was a prospective study including thirty patients who presented with clinical diagnosis of subacromial impingement and referred to perform shoulder MRI at the Radiology Department, Benha University Hospital, in the period between October 2018 and February 2020.

The study was approved by the local research ethical committee at the Faculty of Medicine, Benha University.

Informed oral consent explaining the details of the procedure was obtained from patients prior to inclusion in this study. 
Patients $^{6}$ selection was based on certain inclusion and exclusion criteria.

\section{Inclusion criteria:}

Shoulder pain for more than 3 months with positive clinical tests.

\section{Exclusion criteria:}

History of shoulder surgery, instability disorders, shoulder girdle fracture, radiation therapy to the shoulder, neoplastic lesions and congenital anomalies of the shoulder.

Patients having contraindications to perform MRI (Patients with any electrically, magnetically or mechanically activated implants, pacemaker, cochlear implants or any metallic orthopedic implants) and claustrophobic patients).

\section{All patients were subjected to the} following:

- Clinical assessment: including history taking and clinical examination.

- Radiological investigations: shoulder sonographic and conventional MRI examinations.

- The results obtained by sonographic examination were compared to the results obtained by MR examination which was considered as the gold standard in our study.

\section{$\underline{\text { Methods }}$}

\section{- Ultrasonography examination:}

- Device: The patients were imaged by high resolution ultrasound linear array transducer of 6-12 $\mathrm{MHz}$ of General Electric company (GE) health care model GE LOGIQ P6.

\section{- Technique of examination:}

All rotator cuff tendons were evaluated, but only the changes in the supraspinatus tendon were analyzed in this study because the rotator cuff tears almost always involve the supraspinatus tendon.

While the patient was seated on a backless chair, the following were examined: long head of the biceps tendon, subscapularis, supraspinatus, infraspinatus and teres minor tendons, acromioclavicular joint, subacromial subdeltoid bursa and dynamic examination for subacromial impingement. The detailed ultrasonographic examination was primary described by Gupta \& Robinson ${ }^{18}$.

\section{MRI examination:}

- Device: All patients' shoulders were imaged by closed high field strength system (1.5 Tesla) magnet unit (MagnetomAvanto, Siemens

\section{Healthcare, Erlangen, Germany).}

\section{- Patient Preparation:}

All metallic objects removed from the patient's body or clothes.

Patients were instructed about the importance of being calm with no motion throughout time of examination. 
- Patient position: The patient lied in supine position with the arm at the side in a position of neutral or mild external rotation and the thumb facing up. Shoulder coils were used.

\section{- Imaging planes and pulse sequences:}

Preliminary scout localizers in axial, sagittal and coronal planes were obtained.

- Axial T1-weighted and axial STIR weighted sequences.

- Coronal oblique T1-weighted, coronal oblique T2-weighted, and coronal oblique STIR weighted sequences.

- Sagittal oblique T2-weighted sequences.

\section{Statistical analysis}

Data were collected, revised, coded and entered to the IBM SPSS software (Statistical Package for Social Science, version 23, IBM Corp., USA, 2015). Suitable analysis was done according to the type of data (parametric and non-parametric) obtained for each variable.

Descriptive statistics: as mean, standard deviations and ranges were used for numerical parametric variables. Categorical variables were presented as frequency and percentages.

Analytical statistics: were used to assess the statistical significance of the difference of non-parametric variables by using Chi-square test and/or Fisher exact test when the expected count in any cell found less than 5 .

A P-value of less than 0.05 was considered statistically significant.

Sensitivity, specificity, accuracy, positive predictive value (PPV), and negative predictive value (NPP) of US examinations of different pathologies were calculated.

\section{Results:}

This study included thirty patients, suffer from shoulder pain \&/or limitation of joint movement. The frequency and percentage according to sex in the study population were 18 male patients represented $60 \%$ and 12 female patients represented $40 \%$. Their ages range from 26 years to 68 years (mean age $=45$ \pm 12 years).

The frequency and the percentage of side affection was 19 patients (63\%) and 11 patients $(37 \%)$, for the right and left shoulder joints, respectively.

Figures 1 and 2 show the percentage of affection of shoulders by different pathologies detected by our gold standard MRI.

Table 1 shows that there was no statistically significant difference found between MRI \& US regarding supraspinatus tendon pathology $(\mathrm{P}>0.05)$.

Table 2 shows that there was no statistically significant difference found 
between MRI \& US regarding non-rotator Table 3 shows that there were 11 patients diagnosed to have supraspinatus tendinopathy by US out of 14 patients diagnosed by MRI with subsequent US sensitivity, specificity and accuracy of $78.6 \%, 87.5 \%$ and $83.3 \%$ respectively. Among the other 3 patients diagnosed by MRI to have supraspinatus tendinopathy, 2 of them had normal sonographic appearance of the supraspinatus tendon, while the third diagnosed as partial thickness tear by US.

Regarding supraspinatus partial thickness tears, 10 cases were diagnosed by MRI, only 8 of them could be detected by US, while the other 2 cases were diagnosed to have supraspinatus tendinopathy by US. The US sensitivity, specificity and accuracy for partial thickness tear are $80 \%, 95 \%$ and $90 \%$ respectively.

The all 4 supraspinatus full thickness tears were diagnosed by both ultrasound and MRI with subsequent US sensitivity, specificity, and accuracy of $100 \%$ for each.

Out of 21 cases diagnosed by MRI to have ACJ osteoarthritis, 19 patients were correctly diagnosed by US with subsequent US sensitivity, specificity, and accuracy of $90.5 \%, 100 \%$ and $93.3 \%$ respectively. cuff pathology $(\mathrm{P}>0.05)$.

Out of 13 cases diagnosed by MRI with subacromial bursitis, US correctly diagnosed 12 cases, while diagnosed additional 1 case with bursitis which was negative by MRI, giving the US sensitivity, specificity and accuracy of $92.3 \%, 94.1 \%$ and $93.3 \%$ respectively.

US only diagnosed 16 cases of the 17 cases detected having shoulder joint effusion by MRI with subsequent US sensitivity, specificity, and accuracy of $94.1 \%, 100 \%$ and $96.7 \%$ respectively.

Regarding Biceps tenosynovitis, the US correctly diagnosed the all 5 cases which were detected by MRI, giving the US sensitivity, specificity and accuracy of 100\% for each.

Case 1: A 50 years old female patient complaining of left shoulder pain and limitation of movement for 9 months accentuated on arm elevation, with no history of trauma. (Figure 3).

Case 2: A 59 years old female patient complaining of sever right shoulder pain of chronic duration with inability to fully abduct her arm, with no history of trauma. (Figure $4)$. 
Table 1: Comparison between MRI \& US regarding supraspinatus tendon pathology.

\begin{tabular}{llcccccccc}
\hline \multicolumn{1}{c}{ Supraspinatus tendon pathology } & \multicolumn{2}{c}{ US } & \multicolumn{2}{c}{ MRI } & $\begin{array}{l}\text { Test } \\
\text { value* }\end{array}$ & $\begin{array}{l}\text { P- } \\
\text { value }\end{array}$ & Sig. \\
\hline \multirow{2}{*}{ Tendinopathy } & No. & $\%$ & No. & $\%$ & \\
& Negative & 17 & $56.7 \%$ & 16 & $53.3 \%$ & & & \\
\multirow{2}{*}{ Partial thickness tear } & Positive & 13 & $43.3 \%$ & 14 & $46.7 \%$ & 0.966 & 0.334 & NS \\
& Negative & 21 & $70 \%$ & 20 & $66.7 \%$ & & & \\
\multirow{2}{*}{ Full thickness tear } & Positive & 9 & $30 \%$ & 10 & $33.3 \%$ & 0.577 & 0.564 & NS \\
& Negative & 26 & $86.7 \%$ & 26 & $86.7 \%$ & & & \\
\hline
\end{tabular}

P-value >0.05: Non significant (NS); P-value <0.05: Significant (S); P-value< 0.01: highly significant (HS)

*: Chi-square test and/or Fisher exact test when the expected count in any cell found less than 5

Table 2: Comparison between MRI \& US regarding non rotator cuff pathology.

\begin{tabular}{|c|c|c|c|c|c|c|c|c|}
\hline \multirow[t]{2}{*}{ Non rotator cuff } & \multirow[t]{2}{*}{ pathology } & \multicolumn{2}{|c|}{ US } & \multicolumn{2}{|c|}{ MRI } & \multirow{2}{*}{$\begin{array}{l}\text { Test } \\
\text { value* }\end{array}$} & \multirow{2}{*}{ P-value } & \multirow{2}{*}{ Sig. } \\
\hline & & No. & $\%$ & No. & $\%$ & & & \\
\hline \multirow{2}{*}{ ACJ osteoarthritis } & Negative & 11 & $36.7 \%$ & 9 & $30 \%$ & \multirow{2}{*}{1.414} & \multirow{2}{*}{0.157} & \multirow{2}{*}{ NS } \\
\hline & Positive & 19 & $63.3 \%$ & 21 & $70 \%$ & & & \\
\hline \multirow{2}{*}{ Subacromial bursitis } & Negative & 17 & $56.7 \%$ & 17 & $56.7 \%$ & \multirow{2}{*}{0.000} & \multirow{2}{*}{1.000} & \multirow{2}{*}{ NS } \\
\hline & Positive & 13 & $43.3 \%$ & 13 & $43.3 \%$ & & & \\
\hline \multirow{2}{*}{ Joint effusion } & Negative & 14 & $46.7 \%$ & 13 & $43.3 \%$ & \multirow{2}{*}{1.000} & \multirow{2}{*}{0.317} & \multirow{2}{*}{ NS } \\
\hline & Positive & 16 & $53.3 \%$ & 17 & $56.7 \%$ & & & \\
\hline \multirow{2}{*}{ Biceps tenosynovitis } & Negative & 25 & $83.3 \%$ & 25 & $83.3 \%$ & \multirow{2}{*}{0.000} & \multirow{2}{*}{1.000} & \multirow{2}{*}{ NS } \\
\hline & Positive & 5 & $16.7 \%$ & 5 & $16.7 \%$ & & & \\
\hline
\end{tabular}

*: Chi-square test 
Benha medical journal vol. 38, special issue (Radiology), 2021

Table 3: Diagnostic validity of different shoulder pathological findings by US as referenced by MRI findings.

\begin{tabular}{llllllllll}
\hline Parameter & TP & TN & FP & FN & Sensitivity & Specificity & PPV & NPV & Accuracy \\
\hline $\begin{array}{l}\text { Tendinopathy } \\
\text { Partial thickness }\end{array}$ & 11 & 14 & 2 & 3 & $78.6 \%$ & $87.5 \%$ & $84.6 \%$ & $82.4 \%$ & $83.3 \%$ \\
tear & 19 & 1 & 2 & $80 \%$ & $95 \%$ & $88.9 \%$ & $90.5 \%$ & $90 \%$ \\
Full thickness tear & 4 & 26 & 0 & 0 & $100 \%$ & $100 \%$ & $100 \%$ & $100 \%$ & $100 \%$ \\
$\begin{array}{l}\text { ACJ osteoarthritis } \\
\text { Subacromial }\end{array}$ & 19 & 9 & 0 & 2 & $90.5 \%$ & $100 \%$ & $100 \%$ & $81.8 \%$ & $93.3 \%$ \\
bursitis & 12 & 16 & 1 & 1 & $92.3 \%$ & $94.1 \%$ & $92.3 \%$ & $94.1 \%$ & $93.3 \%$ \\
Joint effusion & 16 & 13 & 0 & 1 & $94.1 \%$ & $100 \%$ & $100 \%$ & $92.9 \%$ & $96.7 \%$ \\
$\begin{array}{l}\text { Biceps } \\
\text { tenosynovitis }\end{array}$ & 5 & 25 & 0 & 0 & $100 \%$ & $100 \%$ & $100 \%$ & $100 \%$ & $100 \%$ \\
\hline
\end{tabular}

TP: True positive; TN: True negative

FP: False positive; FN: False negative

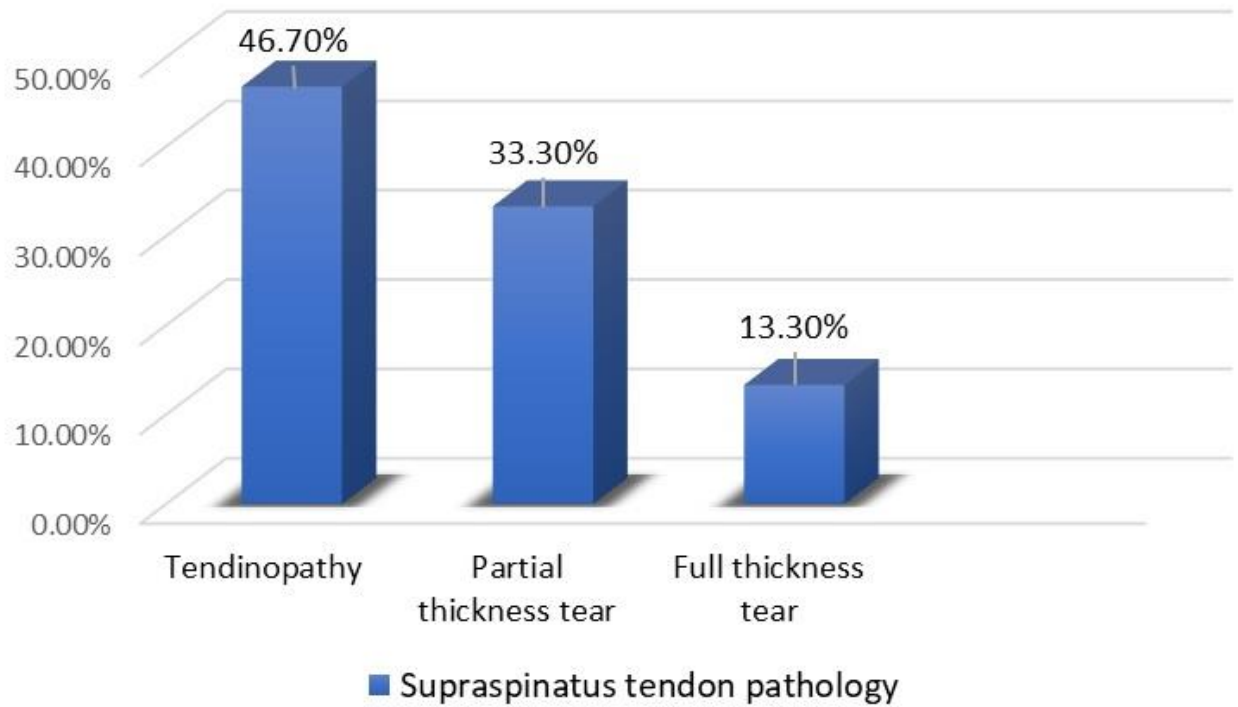

Fig (1): The percentage of supraspinatus tendon pathological findings detected by MRI. 


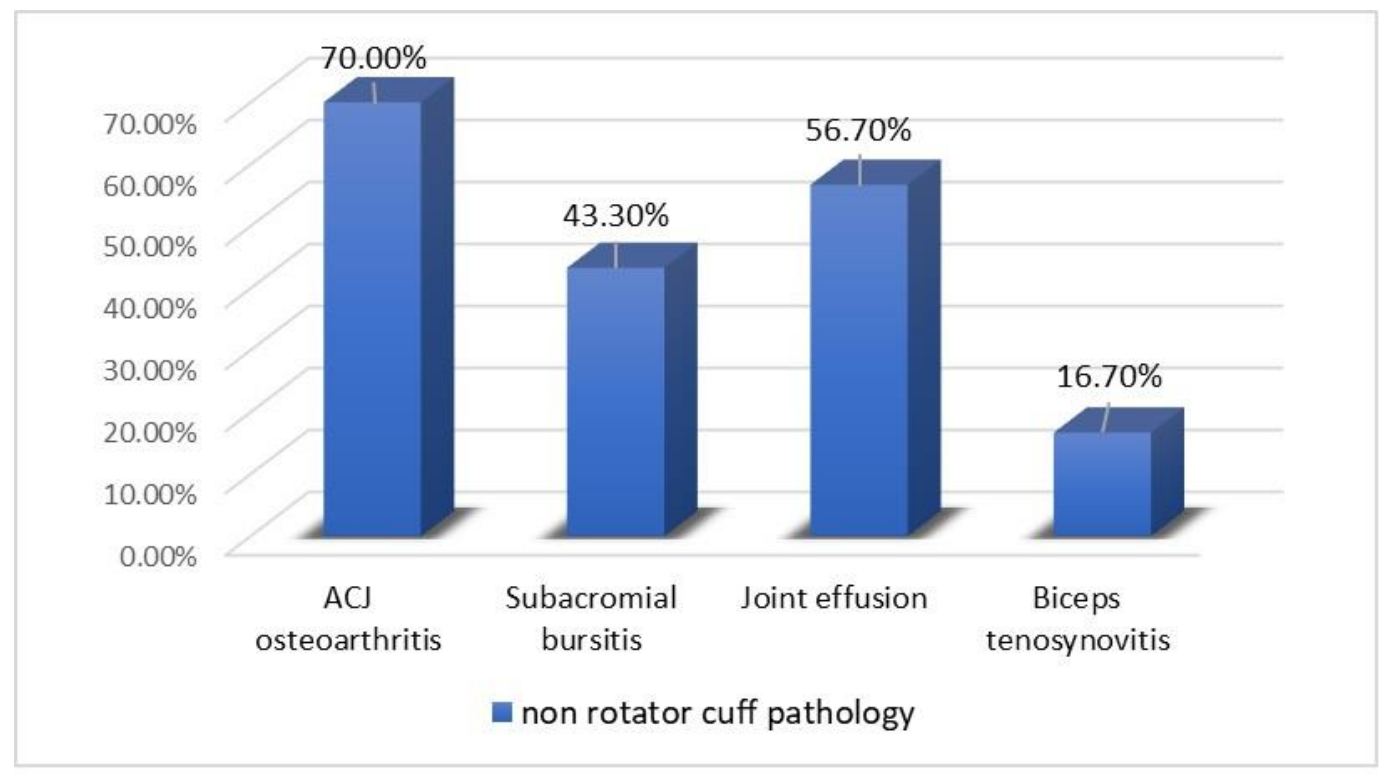

Fig (2): The percentage of non-rotator cuff pathological findings detected by MRI.
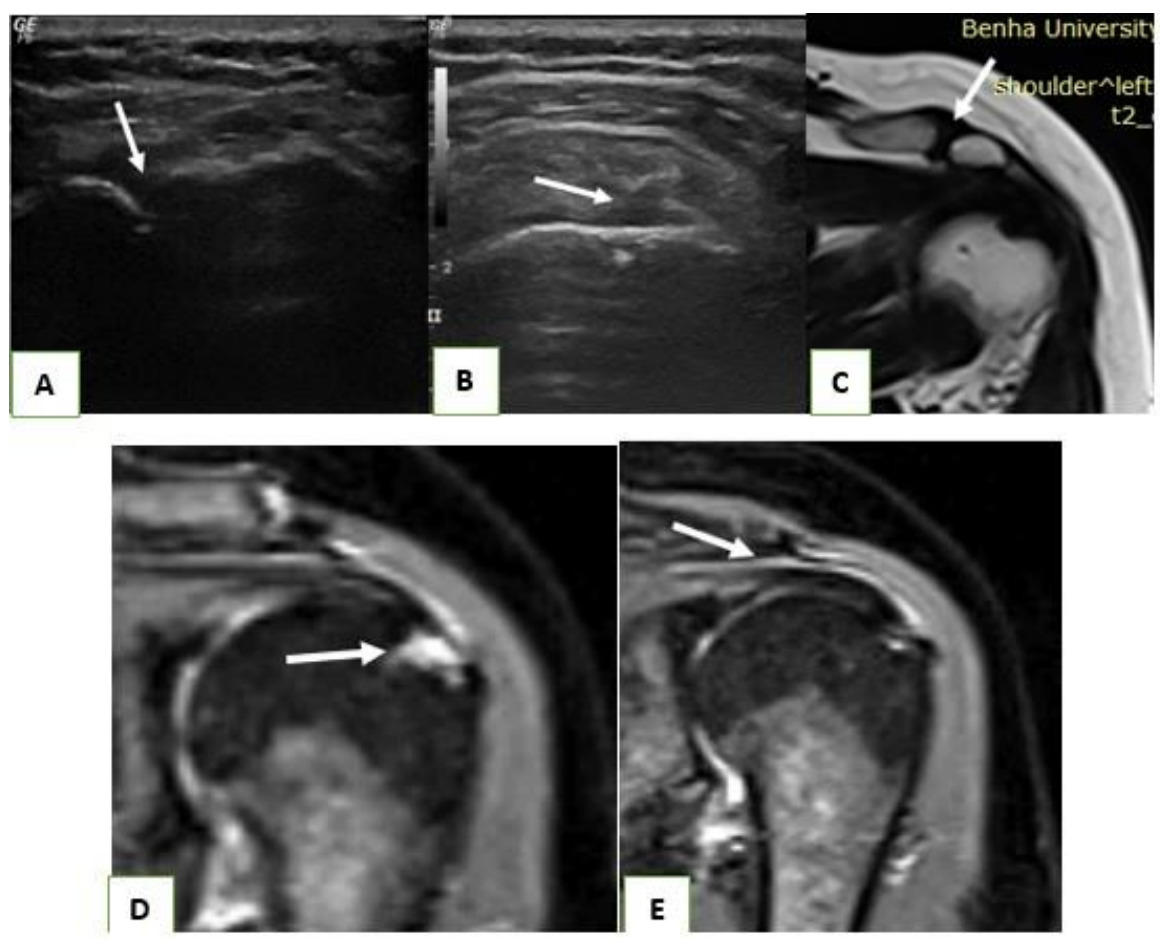

Fig (3): A: US image showed evidence of acromioclavicular osteoarthritis (arrow). B: US image of the shoulder showed articular surface partial thickness tear of the supraspinatus tendon, seen as focal hypoechoic defect at the articular side of the tendon fibers (arrow) with fluid in the subacromial bursa. C: MRI, coronal T2WIs showed acromioclavicular osteoarthritic changes (arrow) and associated acromial offset, both seen obliterating the underlying peritendinous fat planes. D: MRI, coronal STIR WIs: fluid signal at the articular surface of the supraspinatus tendon denoting articular surface partial thickness tear (arrow). E: MRI, coronal STIR WIs: Mild subacromial bursitis (arrow) and mild joint effusion. 


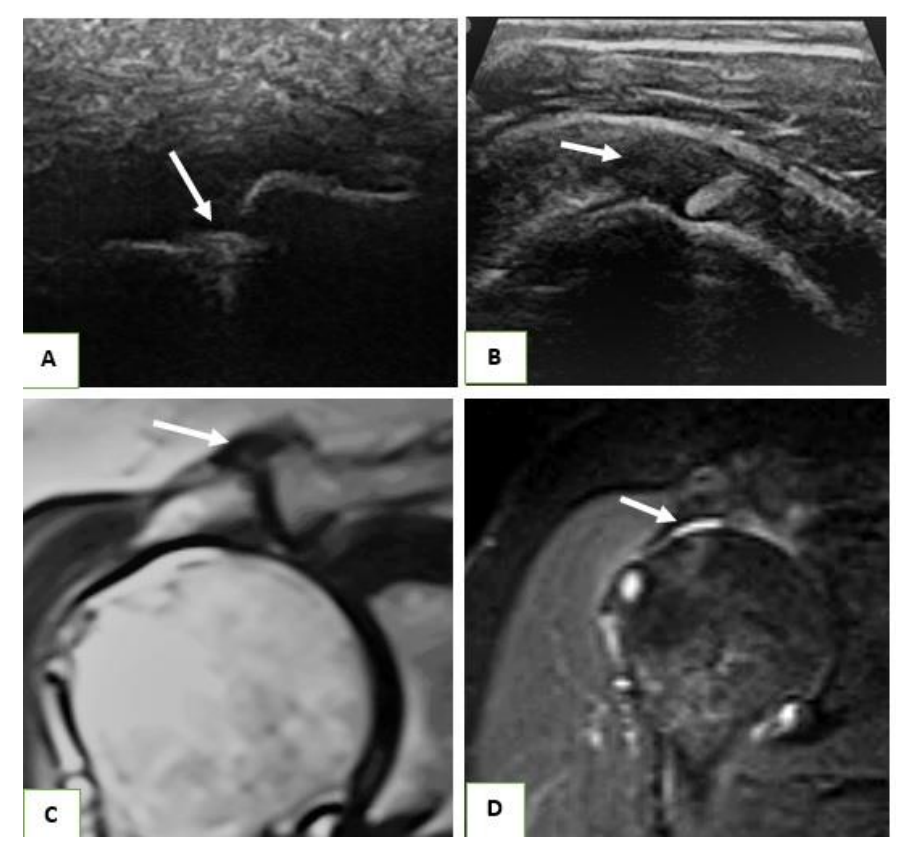

Fig (4): A: US image showed evidence of acromioclavicular osteoarthritis (arrow). B: US image showed hypoechoic defect interrupting the full thickness fibers of the supraspinatus tendon at the insertional site denoting full thickness tear (arrow). C: MRI, coronal T2WIs showed hypertrophic acromioclavicular osteoarthritic changes (arrow) effacing the underlying peritendinous fat planes. D: MRI, coronal STIR WIs showed interrupted continuity of the supraspinatus tendon from its insertional site with fluidfilled gap denoting full thickness tear (arrow) with mild joint effusion.

\section{Discussion:}

Shoulder impingement is the most common shoulder problem; approximately $20 \%$ of people will experience symptoms at some time during their life. This condition developed when the rotator-cuff tendons of the shoulder are overused or injured, causing pain and movement restrictions ${ }^{19}$. It is classified into four types; The subacromial subtype is by far the most common one ${ }^{20}$.

Subacromial impingement syndrome is a result of chronic irritation of the supraspinatus tendon while it passes through the subacromial space, which is the entry underneath the anterior third of the acromion, the coracoacromial ligament and the acromioclavicular joint ${ }^{21}$.

The purpose of the present study was to evaluate and compare the role of dynamic ultrasonography and MR imaging in the detection of different pathological findings encountered in cases of sub acromial impingement, in order to offer a reliable, accurate, highly sensitive and readily available diagnostic tool for these.

In our present study, we had confirmed the fact that MR examination "in cases of subacromial impingement" is invaluable diagnostic modality, that can precisely delineate the anatomic details, the acromial shape, subacromial bursa abnormalities, 
rotator cuff abnormalities including tendinosis, partial-thickness and fullthickness tears as well as degenerative changes of the acromioclavicular joint.

However MRI has several disadvantages; being a static examination that can't reveal the exact relationship between the acromion, humeral head \& intervening soft tissues during active shoulder movement, in addition, its high cost, long examination time \& patient discomfort and fear, besides the questionable availability of this modality added to the general consideration of MRI use.

On other hand, ultrasonography can provide direct real time visualization of the relationship between the acromion, subacromial bursa, supraspinatus tendon and greater tuberosity of the humeral head during active shoulder movement which allows for dynamic scanning.

In the present study, 30 patients were included. These patients had shoulder pain and/or restricted joint movements for more than three months with established clinical diagnosis of subacromial impingement. Cases with history of shoulder dislocation, fractures, surgical intervention or neoplastic lesions were excluded.
All cases were subjected to static/dynamic ultrasonography \& conventional MRI examination. Additional statistical analysis was done to assess the sensitivity of shoulder ultrasound compared to shoulder MRI which is considered as the gold standard diagnostic tool in current practice.

Out of 30 patients included in this study, 18 patients were male representing $60 \%$ while 12 were female patients representing $40 \%$. The age of patients ranged from 26 to 68 years with mean age $45 \pm 12$ years. The right shoulder joints were affected in 19 patients representing $63 \%$ while the rest were left shoulders representing $37 \%$.

This finding is in agreement with a published study, which is a prospective study of MRI and high resolution US in the diagnosis of rotator cuff tears that was performed on 32 patients and revealed higher male incidence $67 \%{ }^{22}$.

It is also in accordance with another study which was performed on 50 patients comparing dynamic US and MRI in diagnosis of shoulder impingement syndrome and revealed higher male incidence $64 \%$ and high incidence of rightsided affection with percentage of $68 \%{ }^{23}$.

In our present study, MRI was used as the gold standard. Out of 30 patients, 28 (93.3\%) 
patients showed supraspinatus tendon pathology either tendinopathy, partial thickness or full thickness tears.

An earlier study also found presence of supraspinatus tendon involvement in around $80 \%$ of cases in their study ${ }^{24}$.

Ultrasound successfully detected 11 cases of supraspinatus tendinopathy, 3 cases were missed and were diagnosed only by MRI, and 2 false positive cases were diagnosed as tendinopathy by ultrasound.

This agreed to some extent with a published study which was revealed ultrasound sensitivity, specificity and accuracy of $83.3 \%, 100 \%$ and $90.9 \%$ respectively. While our study revealed ultrasound sensitivity, specificity and accuracy $78.6 \%, 87.5 \%$ and $83.3 \%$ respectively in the diagnosis of supraspinatus tendinopathy ${ }^{23}$.

Our results are also in agreement with a meta-analysis which was done to assess the diagnostic accuracy of ultrasound, MRI and MR arthrography in the characterization of rotator cuff disorders with analysis of 82 articles, and showed that mean sensitivity and specificity of US in detection of supraspinatus tendinopathy $79 \%$ (range from $63 \%$ to $91 \%$ ) and $94 \%$ (range from $86 \%$ to $99 \%$ ) respectively 15 .

The US correctly picked supraspinatus tendon partial thickness tears in 8 patients. 2 patients with partial thickness tears of supraspinatus on MRI were misdiagnosed to have tendinopathy on US.

Our results are in accordance with an earlier study done on 50 patients, to evaluate and compare ultrasonography to MRI in diagnosis of rotator cuff pathologies and tear, with a sensitivity of $78.04 \%$ and specificity of $89.47 \%^{25}$, in comparison to our study which revealed $80 \%$ sensitivity and $95 \%$ specificity for partial thickness tear.

The level of sensitivity and specificity seen in our study closely resembles to that study which reported a sensitivity of $79 \%$ and specificity of $94 \%$ in detection of partial thickness tears ${ }^{26}$.

These results are not in accordance with another study that reported the very high sensitivity (about 100\%) of ultrasonography in detection of different types of partialthickness rotator cuff tears ${ }^{27}$.

In the present study, 4 cases had supraspinatus full-thickness tear were detected by both MRI and ultrasonography with subsequent US sensitivity, specificity, PPV, NPV, and accuracy of $100 \%$ for each.

This agreed with a study that was performed on 30 patients with suspected rotator cuff disorders who were subjected to both US and MRI investigations, correlating between 
their results. It reported $100 \%$ sensitivity, $96.4 \%$ specificity and an accuracy of $100 \%$ in identifying full-thickness tears ${ }^{28}$.

Our results are also in accordance with a published study that was performed on 190 consecutive shoulders in 185 patients to compare the US findings with arthroscopy and revealed US sensitivity $100 \%$, specificity 91\%, PPV 91\%, NPV 100\%, and accuracy $95 \%$ in detection of full-thickness tears $^{29}$.

Our results are also comparable and coincide with a previous study which reported sensitivity and accuracy of US in detecting full-thickness tears of $92.9 \%$ and $89 \%$, respectively ${ }^{30}$.

Acromioclavicular joint osteoarthritis was the most observed non rotator cuff related pathology in our study. 21 patients were diagnosed by MRI, while only 19 cases were detected by US giving it a sensitivity of $90.5 \%$ and specificity of $100 \%$.

This findings is similar to that of a previous study who showed accepted accuracy of dynamic ultrasonography in detection of acromioclavicular joint osteoarthritic changes compared to MRI with a sensitivity of 94.7 and specificity of $100 \%{ }^{23}$.
Our results are also comparable and coincide with another study which was performed on 60 patients to compare the role of US with MRI in shoulder injuries, and reported the value of dynamic ultrasonography in direct visualization of the rotator cuff tendon injury by acromioclavicular joint degenerative changes with a sensitivity of $80 \%$ and specificity of $95 \%{ }^{31}$.

The current study showed high diagnostic sensitivity of ultrasonography in detection of fluid in both joint space and bursa, namely the sub acromial bursa, that is a common finding in cases of subacromial impingement, not only as a causative factor in cases of extrinsic impingement and as an association in most cases of acromioclavicular osteoarthritis, rotator cuff tendinosis and rotator cuff tears, but also as a predictive value for full thickness supraspinatus tears.

In this study, 12 cases showed evidences of subacromial bursitis with bursal fluid distension by ultrasonography, while MRI had detected 13 cases, giving the US sensitivity and specificity of $92.3 \%$ and $94.1 \%$. It was noticed that the cases missed by US showed very minimal bursal effusion. Regarding joint effusion, among the cases of the study there were 17 cases having joint 
effusion by MRI, 1 of them missed by ultrasonography with subsequent US sensitivity and specificity of $94.1 \%$ and 100 $\%$ respectively.

This is in agreement with a published study, who reported sensitivity and specificity of $93.3 \%$ and $100 \%$ respectively for subacromial bursitis, and sensitivity and specificity of $94.2 \%$ and $100 \%$ respectively for joint effusion ${ }^{23}$.

This disagreeing with another study who showed US sensitivity of $44.4 \%$ in detection of subacromial-subdeltoid bursitis when compared with MRI ${ }^{31}$.

In this study, 5 cases detected by MRI could be also detected by ultrasonography to have biceps tenosynovitis, giving the US sensitivity, specificity, and accuracy of $100 \%$ for each.

This is in accordance with a study who reported high diagnostic value of US with sensitivity, specificity, and accuracy of $100 \%$ for each in detection of bicep tenosynovitis $^{23}$.

Our result is also comparable and coincide with an earlier study who showed $100 \%$ sensitivity in detection of biceps tenosynovitis $^{31}$.

\section{Conclusion:}

US is comparable to MRI in evaluation of the shoulder impingement and rotator cuff disorders. It proved to have high sensitivity and specificity for full thickness tears with relatively less sensitivity and specificity in detection of partial thickness tears. US can be used as the first line imaging modality in patients presenting with shoulder pain. MRI can be used in situations where the diagnosis is ambiguous on US. A well performed ultrasound examination in most cases obviates the need for more diagnostic tests like arthrography and MRI examinations.

Ultrasonography being non-invasive, nonionizing, widely available combined with low cost with advantage of dynamic real time assessment, may serve as the most costeffective imaging method for screening of shoulder impingement syndrome and rotator cuff disorders.

\section{References}

1. Ardic F, Kahraman Y, Kacar M, Kahraman MC, Findikoglu G, Yorgancioglu ZR. Shoulder impingement syndrome: Relationships between clinical, functional, and radiologic findings. Am J Phys Med Rehabil. 2006;85(1):53-60. doi:10.1097/01.phm.0000179518.85484.53

2. Watts AR, Williams B, Kim SW, Bramwell DC, Krishnan J. Shoulder impingement syndrome: a systematic review of clinical trial participant selection criteria. Shoulder Elb. 2017;9(1):31-41. 
doi:10.1177/1758573216663201

3. Harrison AK, Flatow EL. Subacromial impingement syndrome. JAAOS-Journal Am Acad Orthop Surg. 2011;19(11):701-708.

4. Williams R, Morton DG. Knowledge of practising radiographers of the supraspinatus outlet projection for shoulder impingement syndrome in the Eastern Cape, South Africa. Radiography. 2016;22(2):e81e85. doi:10.1016/j.radi.2015.10.006

5. Bigliani LU, Levine WN. Current concepts reviewsubacromial impingement syndrome. JBJS. 1997;79(12):1854-1868.

6. Dietrich TJ, Jonczy M, Buck FM, Sutter R, Puskas GJ, Pfirrmann CWA. Ultrasound of the coracoacromial ligament in asymptomatic volunteers and patients with shoulder impingement. Acta radiol. 2016;57(8):971-977. doi:10.1177/0284185115610930

7. Neer CS. Impingement lesions. Clin Orthop Relat Res. 1983;173:70-77.

8. Seeger LL, Gold RH, Bassett LW, Ellman H. Shoulder impingement syndrome: MR findings in 53 shoulders. Am J Roentgenol. 1987;150(2):343347. doi:10.2214/ajr.150.2.343

9. Neer CS, Surgery J. Anterior Acromioplasty for the Chronic Impingement Syndrome in the JBJS Classics Anterior Acromioplasty for the Chronic Impingement Syndrome in the Shoulder. $J$ Bone $J t$ Surg Am Vol. 1972;54(6):41-50. doi:10.2106/JBJS.8706.cl

10. Karim K, Sah RK, Rasheed A, Awais SM. Functional Improvement after Sub-Acromial Corticosteroid Injection in Patient with Shoulder Impingement Syndrome. Ann King Edward Med Univ. 2016;22(2).
11. Naqvi G, Jadaan M, Harrington P. Accuracy of ultrasonography and magnetic resonance imaging for detection of full thickness rotator cuff tears. Int J Shoulder Surg. 2009;3(4):94. doi:10.4103/0973-6042.63218

12. Seibold CJ, Mallisee TA, Erickson SJ, Boynton MD, Raasch WG, Timins ME. Rotator cuff: Evaluation with US and MR imaging. Radiographics. 1999;19(3):685-705. doi:10.1148/radiographics.19.3.g99ma03685

13. Wiener SN, Seitz WH. Sonography of the shoulder in patients with tears of the rotator cuff: Accuracy and value for selecting surgical options. Am J Roentgenol. 1993;160(1):103-107. doi:10.2214/ajr.160.1.8416605

14. Chang CY, Wang SF, Chiou HJ, Ma HL, Sun YC, Wu HD. Comparison of shoulder ultrasound and MR imaging in diagnosing full-thickness rotator cuff tears. Clin Imaging. 2002;26(1):50-54. doi:10.1016/S0899-7071(01)00323-0

15. Roy JS, BraÃn C, Leblond J, Desmeules F, Dionne CE, MacDermid JC, et al. Diagnostic accuracy of ultrasonography, MRI and MR arthrography in the characterisation of rotator cuff disorders: A systematic review and meta-analysis. Br J Sports Med. 2015;49(20):1316-1328. doi:10.1136/bjsports-2014-094148

16. Lenza M, Buchbinder $\mathrm{R}$, Takwoingi $\mathrm{Y}$, Johnston R V., Hanchard NCA, Faloppa F. Magnetic resonance imaging, magnetic resonance arthrography and ultrasonography for assessing rotator cuff tears in people with shoulder pain for whom surgery is being considered. Cochrane Database Syst Rev. 2013;2013(9). doi:10.1002/14651858.CD009020.pub2

17. Frei R, Chládek P, Trc T, Kopecný Z, 
Kautzner J. Arthroscopic evaluation of ultrasonography and magnetic resonance imaging for diagnosis of rotator cuff tear. Ortop Traumatol Rehabil. 2008;10(2):111-114.

18. Gupta H, Robinson P. Normal shoulder ultrasound: Anatomy and technique. Semin Musculoskelet Radiol. 2015;19(3):203-211. doi:10.1055/s-0035-1549315

19. Bury J, West M, Chamorro-Moriana G, Littlewood C. Effectiveness of scapula-focused approaches in patients with rotator cuff related shoulder pain: a systematic review and metaanalysis. Man Ther. 2016;25:35-42.

20. Garving C, Jakob S, Bauer I, Nadjar R, Brunner UH. Impingement syndrome of the shoulder. Dtsch Arztebl Int. 2017;114(45):765-776. doi:10.3238/arztebl.2017.0765

21. Manzoor I, Bacha R, Gilani S, Liaqat M. The Role of Ultrasound in Shoulder Impingement Syndrome and Rotator Cuff Tear. Ann Orthop Trauma Rehabil. 2019;2(1):126.

22. Chauhan NS, Ahluwalia A, Sharma YP, Thakur L. A prospective comparative study of high resolution ultrasound and MRI in the diagnosis of rotator cuff tears in a tertiary hospital of North India. Polish J Radiol. 2016;81:491.

23. El-Shewi IEHAF, El Azizy HM, Gadalla AAEFH. Role of dynamic ultrasound versus MRI in diagnosis and assessment of shoulder impingement syndrome. Egypt J Radiol Nucl Med. 2019;50(1). doi:10.1186/s43055-019-0107-7

24. Zlatkin MB. MRI of the Shoulder. Lippincott
Williams \& Wilkins; 2003.

25. Bashir S, Firdose SR, Kamal Y, Khan HA, Arora M, Gul S, et al. Correlation between high resolution ultrasonography and MRI in rotator cuff tear diagnosis. Int J Heal Sci Res. 2014;4(4):103112.

26. Cullen DM, Breidahl WH, Janes GC. Diagnostic accuracy of shoulder ultrasound performed by a single operator. Australas Radiol. 2007;51(3):226-229.

27. Melanie F, Grey S. Sonography of suprapinatus tears. AJR. 2005;184:180-184.

28. Naganna HP, Rangaswamy SM, Puttaraju NC. O riginal R esearch A rticle Study of Rotator Cuff Disorders by Ultrasound with Magnetic Resonance Imaging Correlation. Int J Contemp Med Surg Radiol. 2018;3(1):70-75.

29. Milosavljevic J, Elvin A, Rahme $\mathrm{H}$. Ultrasonography of the rotator cuff: a comparison with arthroscopy in one-hundred-and-ninety consecutive cases. Acta radiol. 2005;46(8):858865.

30. Chen D-Y, Lan HH-C, Lai K-L, Chen H-H, Chen Y-M, Chen C-P. Diagnostic utility of US for detecting rotator cuff tears in rheumatoid arthritis patients: comparison with magnetic resonance imaging. J Med Ultrasound. 2014;22(4):200-206.

31. Rao A. Role of Ultrasound in Evaluation of Shoulder Injuries: A Comparative Study of Ultrasound and MRI Abhinav Pratap Singh, Anuradha Rao, Siddalinga Devaru. 2017;(May). doi:10.7860/IJARS/2017/24542

To cite this article: Medhat M. Refaat, Ahmed Torky, Waleed Salah El Deen, Sally Soliman Comparing Efficacy of Shoulder Ultrasound and Magnetic Resonance Imaging in Shoulder Impingement. BMFJ 2021; 38 (Radiology): 112-127. DOI: 10.21608/bmfj.2020.48984.1339 